Received: 10 November 2018

Accepted: 13 March 2019

Published online: 18 April 2019

\section{Maternal DCAF13 Regulates Chromatin Tightness to Contribute to Embryonic Development}

\begin{abstract}
Maternal-zygotic transition (MZT) is critical for the developmental control handed from maternal products to newly synthesized zygotic genome in the earliest stage of embryogenesis. However, the spatiotemporal dynamic regulation of MZT by maternal factors is largely unknown. Here, we reported a novel maternal factor, DCAF13, which was highly expressed in growing oocyte nucleolus and had key maternal effects on oocyte and zygotic chromatin tightness during maternal to zygotic transition. DCAF13 specifically deleted in oocytes resulted in loose chromatin structure in fully grown germinal vesicle oocytes. Despite normal nuclear maturation in maternal DCAF13-deleted oocytes, the chromosomes at MII stage were not properly condensed. Consequently, the nuclear and nucleolar structure reorganized abnormally, and transcription was inactive in zygotic embryos. RNA-seq analysis of MII oocytes and 2-cell embryos demonstrated that the transcriptomes between knockout and control oocyte were similar, but the maternal DCAF13 deleted two-cell embryos showed a significant decrease in transcription. In addition, the maternal DCAF13-deleted embryos displayed arrest at the two-cell stage, which could not be rescued by injecting flag-Dcaf13 mRNA in the zygote. This revealed that DCAF13 was a unique maternal effect factor regulating the nucleolus.
\end{abstract}

In vertebrates, maternal-zygotic transition (MZT) is not a one-stage process, but a process that is active from fertilization to the step when the zygotic genome is fully activated, and maternal mRNAs are completely degraded ${ }^{1}$. Eventually, successful zygotic genome activation (ZGA) is required for further embryo development, failure of which causes growth arrest. Prior to zygotic genome activation, the initiation of zygotic development and nuclear reorganization are under the control of maternal factors ${ }^{2,3}$, which occurs at the late two-cell stage in mice and four-eight-cell stage in humans ${ }^{4}$.

The morphology and structure of the nucleus need to be reformed to adjust to the transcription during $\mathrm{MZT}^{5}$. During the period of oocyte growing, the chromatin is less condensed and not confined around the nucleolus, termed the non-surrounded nucleolus (NSN) configuration ${ }^{6}$. However, when it comes to fully grown oocytes, there is a shutdown in new transcript synthesis, and the chromatin is rather condensed and particularly confined around the nucleolus, termed the surrounded nucleolus (SN) configuration ${ }^{7}$. Although the chromatin is condensed during meiosis, once fertilization, the chromatin structure of pronucleus (PN) in zygote turns to be extremely loose, leading to the whole genome to be poorly transcribed in the zygote ${ }^{8}$. However, the detailed regulatory mechanism behind this is largely unclear.

The maternal nucleus structure or factors affect ZGA ${ }^{9}$. Embryos derived from denucleated oocytes fail to develop beyond the two-cell stage, suggesting that the oocyte nucleus has a pivotal role in early embryonic development $^{10}$. These studies suggest that the nucleus/nucleolus morphology and chromatin configuration need to be changed to adapt to the gradually recovered transcription during MZT.

DCAF13 is one of the major substrate adaptors of the DDB1/CUL4 complex, which is an E3 ligase ${ }^{11}$. In our previous studies, Dcaf13 knockout embryos are arrested at the morula stage, accompanied with increased H3K9me3 level in blastomeres of 8-cell embryos. Furthermore, we show that DCAF13 bridges ubiquitin E3 ligase CRL4 to histone methyltransferase SUV39H1, and target this enzyme for polyubiquitination and proteasomal degradation. As a result, CRL4-DCAF13 facilitates H3K9me3 removal and zygotic gene expression in preimplantation embryos ${ }^{12}$. In order to explore the role of maternal DCAF13 in oocytes, we specifically deleted Dcaf 13 in developing oocytes, as early as the primordial follicle stage using Cre transgene driven by Gdf9 promoter

${ }^{1}$ Laboratory of Medical Genetics, Harbin Medical University, Harbin, China. ${ }^{2}$ Life Sciences Institute, Zhejiang University, Hangzhou, 310058, China. Correspondence and requests for materials should be addressed toY.J. (email: jinyan@ems. hrbmu.edu.cn) or H.-Y.F. (email: hyfan@zju.edu.cn) 
(Gdf9-Cre). In this mouse model, the majority of the oocytes fail to develop to the preovulatory stage and became apoptotic. The female mice are infertile due to premature ovarian failure. Mechanistically, DCAF13 binds with the nucleolar protein fibrillarin and is involved in the processing of $18 \mathrm{~S}$ rRNA during oocyte growth ${ }^{13}$.

However, the function of maternal DCAF13 in MZT is still largely unclear. In this study, we generated conditional knockout mice with a deletion of Dcaf 13 in oocytes from primary follicles using Zp3-Cre, which was a powerful model to elucidate the function of maternal DCAF13 during the early development of the zygotes ${ }^{14}$. We found that maternal DCAF13 participated in the zygotic nucleus/nucleolus reformation and function and was involved in the switch of chromatin tightness during MZT. By DCAF13 maternally depletion, the zygote cannot activate its genome transcription, render the embryo development, and arrest at two-cell stage. Together, our results pointed out DCAF13 as a new maternal factor that regulated chromatin tightness and affected the zygotic nucleus/nucleolus reformation and ZGA.

\section{Results}

DCAF13 is essential for female fertility but not for oocyte meiotic maturation. In mouse oocytes, DCAF13 expression decreases during the transition from mature to climacteric stage. In a previous study, we found that DCAF13 was localized in the nucleolus and was essential for 18S rRNA transcription and nucleolus function in growing oocytes ${ }^{13}$. However, whether DCAF13 is essential for female fertility is unclear. We used the growing oocyte-specific deletion strategy by crossing Zp3-Cre transgenic mice with the Dcaf $13^{\text {fl/fl }}$ mice, in which the Dcaf13 gene was deleted specifically in oocytes from the primary follicle stage. Dcaf $13^{f l f f} ; Z p 3$ Cre (Dcaf $13^{o o-l-}$ ) females were then mated with wild-type males. Strikingly, we found that progeny was barely obtained compared to Dcaf $13^{f l / f l}$ females. This indicated that Dcaf $13^{f l f l} ; Z p 3-C r e$ female were sterile (Fig. 1a). To determine whether the reasonof sterility was the defect in oocytes, we isolated fully grown GV stage oocytes from $D c a f 13^{f l / f l}$ and Dcaf $13^{f l f l} ; Z p 3-C r e$ females and investigated the in vitro oocyte meiotic maturation process. We found that the Dcaf1 $3^{\text {fllfl }} ; Z p 3$-Cre oocytes did not show any defect in GVBD or PB1 emission (Fig. 1b). To further confirm the quality of oocytes, we also detected several rRNA level (18S rRNA, 28S rRNA, and 5.8S rRNA) which were the products of pre-rRNA processing. The results showed that rRNA processing in oocytes was not significantly affected in these fully-grown oocytes collected from young females of Dcafl $3^{\text {fllfl }} ; \mathrm{Zp} 3$-Cre mice (see Supplementary Fig. S2).

DCAF13 deletion results in loose chromatin structure in fully grown GV oocytes. At the fully grown GV stage, the transcription of the oocytes was inactive, and the chromatin appeared as a condensed perinucleolar ring (SN oocytes). However, in Dcaf $13^{f l f l}$;Zp3-Cre GV stage oocytes, the SN oocytes were notably less than those in Dcaf $13^{f l f l} \mathrm{GV}$ stage oocytes, and most of their chromatin was dispersed (NSN oocytes), indicating that DCAF13 regulated the chromatin configuration. To confirm the hypothesis of DCAF13 function in the regulation of chromatin configuration, we performed a DNase I sensitivity assay to test the tightness of chromatin. If the chromatin structure was loose, it was easily exposed to DNase I and would show higher sensitivity. As we predicted, when the DNase I concentration increased, Dcaf $13^{f l f f} ; Z p 3-C r e$ oocytes showed clear signals of DNA damage by increased levels of terminal deoxynucleotidyl transferase (TdT)-mediated deoxyuridine triphosphate (dUTP) nick-end labeling (Fig. 1c-e). Therefore, we concluded that the chromatin tightness level decreased in DCAF13-deleted oocytes.

DCAF13-maternally depleted embryos display abnormal chromatin tightness level and nuclear configuration. Owing to the chromatin configuration defects observed following the deletion of DCAF13 in oocytes, we next sought to study whether this defect also perturbed the reorganization of the nucleus and nucleolus after fertilization. First, we investigated the chromatin tightness of the WT and Dcaf $13^{-1+}$ embryos. With the increased concentration of DNase I, we examined the signals of DNA damage and quantified the relative fluorescence intensity. Upon treatment with $0.1 \mathrm{U} / \mu \mathrm{L}$ DNase I, Dcaf $13^{-1+}$ two-cell embryos showed less DNA damage compared to control two-cell embryos (Fig. 2a,b).

To address the interconnection between nuclear morphology and chromatin tightness, we investigated the nuclear morphology of them. We found that abnormal nuclear and nucleolus morphology was observed in Dcaf13 ${ }^{-1+}$ two-cell embryos (Fig. 2c). To measure this change, we set up a method to measure the ratio of minor-major axis. In Dcaf $13^{+1+}$ two-cell embryos, the nucleus was close to roundness, and the ratio was close to 1 . But in Dcaf13 $1+$ two-cell embryos, the nucleus morphology was abnormal, and the ratio was deviated to 1 (Fig. 2d). The most striking effect we detected is the morphology of the nucleoli. We found that the size of the nucleoli was not uniform in Dcaf $13^{-1+}$ two-cell embryos. By classifying the nucleoli based on area scores, the number and percentage of nucleoli which had abnormal size was elevated in Dcaf $13^{-1+}$ two-cell embryos (Fig. 2e). In both, chromatin tightness levels were abnormally elevated and nucleus reorganization was failed in DCAF13-maternally depleted two-cell embryos.

Depletion of maternal DCAF13 results in developmental arrest at the two-cell stage. Next, we mated Dcaf $13^{f l / l}$ and Dcaf $13^{f l f f} ; Z p 3$-Cre females with wild-type males, and then embryos were isolated on embryonic day 2 (E2) (Fig. 2f). Surprisingly, we found that most of the Dcaf1 $3^{-1+}$ embryos were arrested at the two-cell stage, and the proportion of Dcaf $13^{-1+}$ four-cell stage embryos recovered ( 20\%) was notably lower than that obtained with Dcaf $13^{+l+}$ embryos (100\%) (Fig. 2g). To assess the developmental fate of Dcaf $13^{-l+}$ two-cell embryos and surviving Dcaf $13^{-1+}$ four-cell embryos, we kept culturing and monitoring them in vitro. After $48 \mathrm{~h}$ in culture, all the surviving Dcaf $13^{-1+}$ embryos were found to be at the blastocyst stage, like Dcaf $13^{+1+}$ embryos, but two-cell embryos were confirmed arrested not developmental delayed (Fig. 2f,g). 

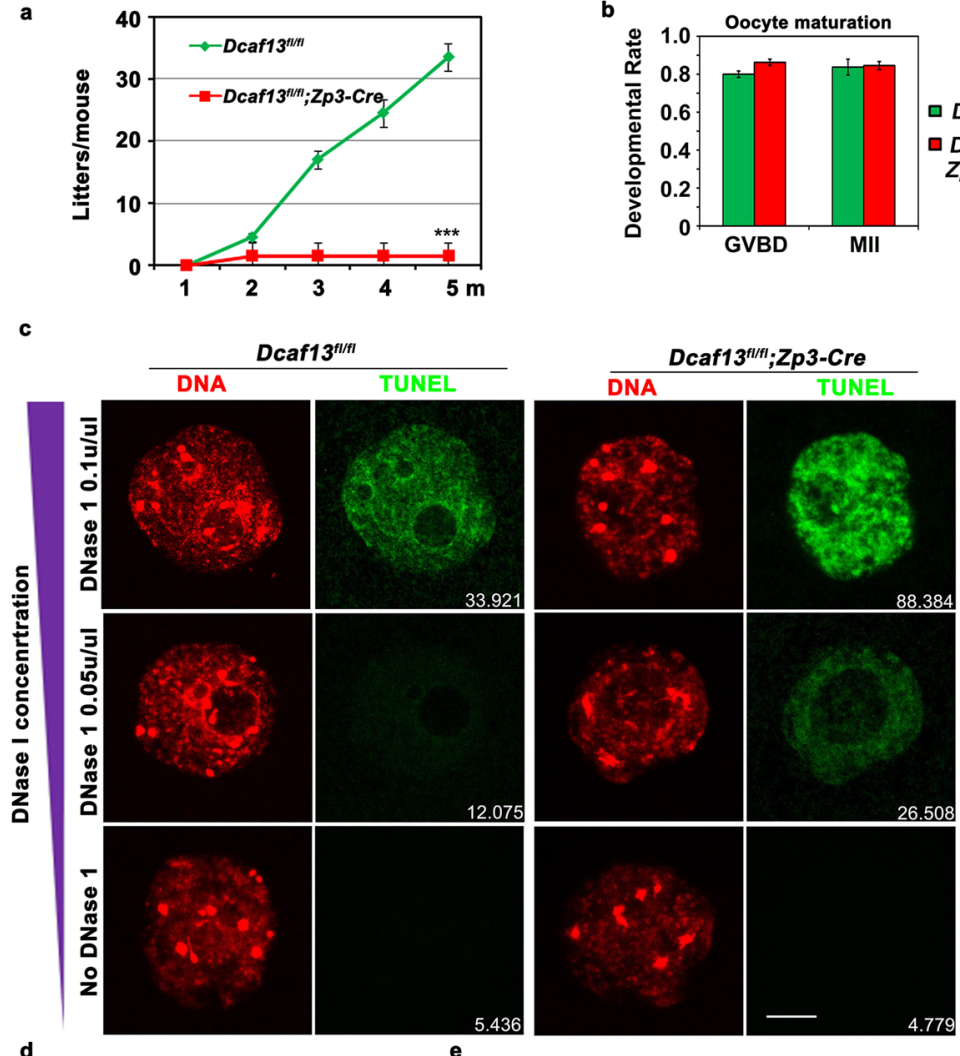

d
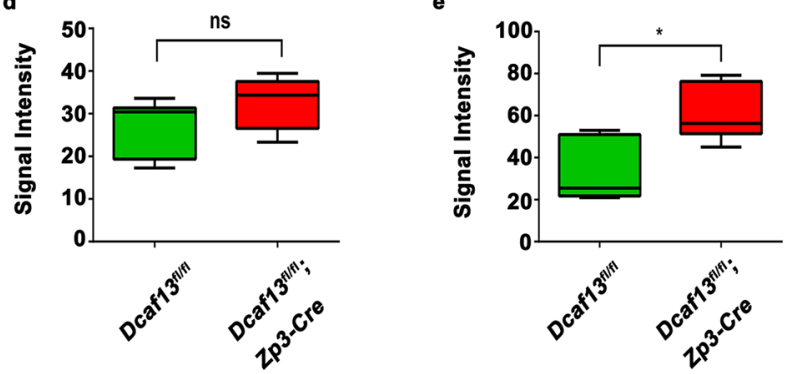

Figure 1. DCAF13 is essential for female fertility but not for oocyte meiotic maturation, and deletion of DCAF13 results in loose chromatin structure in fully grown germinal vesicle (GV) oocytes. (a) Cumulative numbers of pups per female (Dcaf1 $3^{f l f l}$ and Dcaf1 $\left.3^{\text {fllfl }} ; \mathrm{Zp} 3-\mathrm{Cre}\right)(\mathrm{n} \geq 5)$. Error bars indicated standard error of the mean (SEM). *** to $p<0.001$. (b) Rates of GVBD and PB emission of Dcaf1 $3^{\text {fllfl }}$ and Dcaf13 fllfl:Zp3-Cre oocytes cultured in vitro. Scale bar, $100 \mu \mathrm{m}$. Dcaf1 $3^{f l f l}(\mathrm{n}=75)$, Dcaf1 $3^{\text {flffl }} ; \mathrm{Zp} 3-C r e(\mathrm{n}=50)$. (c) Lack of DCAF13 in oocytes led to increased DNase I accessibility. Scale bar, $25 \mu \mathrm{m}$. (d,e) The positive TUNEL signal of 0.05 $\mathrm{U} / \mu \mathrm{L}$ and $0.1 \mathrm{U} / \mu \mathrm{L}$ DNase I is indicated. $*$ to $p<0.05, \mathrm{~ns}$ to $p>0.05 .0 .1 \mathrm{U} / \mu \mathrm{L}$ DNase I: Dcaf1 $3^{\text {flfl }}(\mathrm{n}=10)$, Dcaf1 $3^{\text {fllfl}} ; Z p 3-C r e(\mathrm{n}=12) .0 .05 \mathrm{U} / \mu \mathrm{L}$ Dcaf13 $3^{\text {flfl }}(\mathrm{n}=12)$, Dcaf13 ${ }^{\text {fllfl }} ; \mathrm{Zp} 3-C r e(\mathrm{n}=12)$.

To determine if the embryo arrest at the two-cell stage was caused by maternal DCAF13 deletion, we compensated DCAF13 in Dcaf13 ${ }^{-1+}$ zygotes by injecting Flag-Dcaf13 mRNA. As shown in Fig. 2h, 75.5\% embryos compensated with DCAF13 (in Dcaf $13^{-1+}$ ) zygotes were found to be significantly blocked at the two-cell stage compared to $9 \%$ in Dcaf13 $3^{+1+}$ embryos. Minor rescues were found with Flag-Dcaf13 mRNA injection for Dcaf $13^{-1+}$ embryos arrested at the two-cell stage, although exogenous FLAG-DCAF13 was effectively expressed at the 2-cell stage (Fig. 2h). Uncropped scans of the Western blot results of DCAF13 expression was provided in Supplementary Fig. S1. Taken together, the maternal DCAF13 was required for zygotes to proceed beyond the two-cell stage.

Maternal DCAF13 affects zygotic genome activation by the two-cell stage. After fertilization, new transcripts need to be generated along with protein synthesis corresponding to $Z G A^{15}$. This progress mainly appears in the late two-cell stage. Therefore, we chose fertilized 54-h embryos (late two-cell stage) to detect the transcription levels. We performed EU staining and immunofluorescence detection of RNA Polymerase II CTD phospho-Ser2 (PolIISer2P), a form of active transcription. Of note, significant differences were detected between Dcaf $13^{+l+}$ and Dcaf $13^{-l+}$ two-cell embryos, and transcription activity decreased by more than half in Dcaf $13^{-1+}$ two-cell embryos (Fig. 3a). To assess the whole transcriptome changes affected by the lack of maternal DCAF13, 

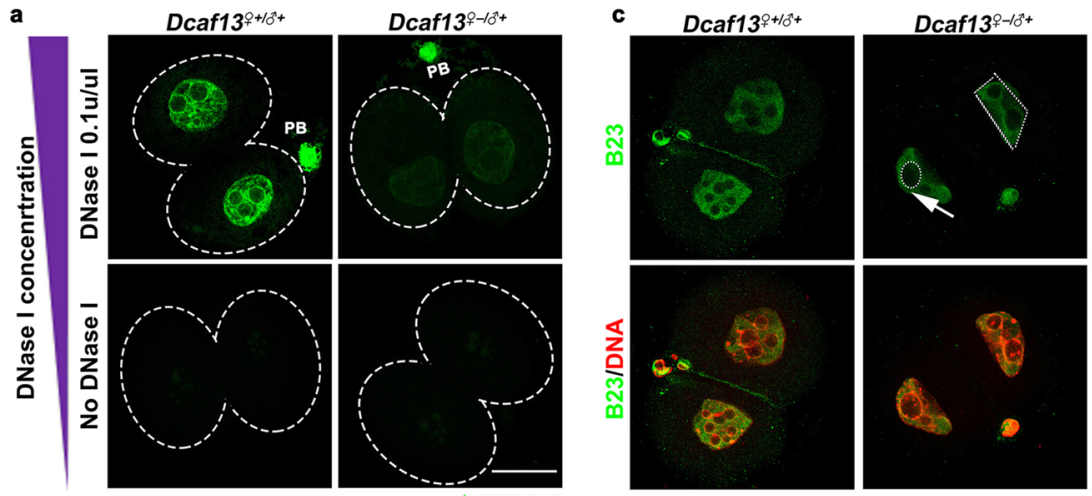

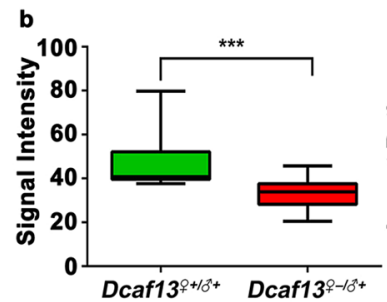

d

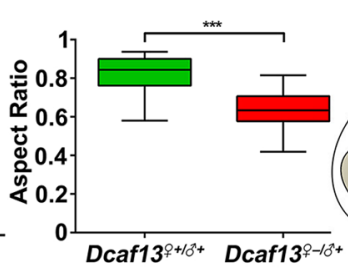

+12 h culture
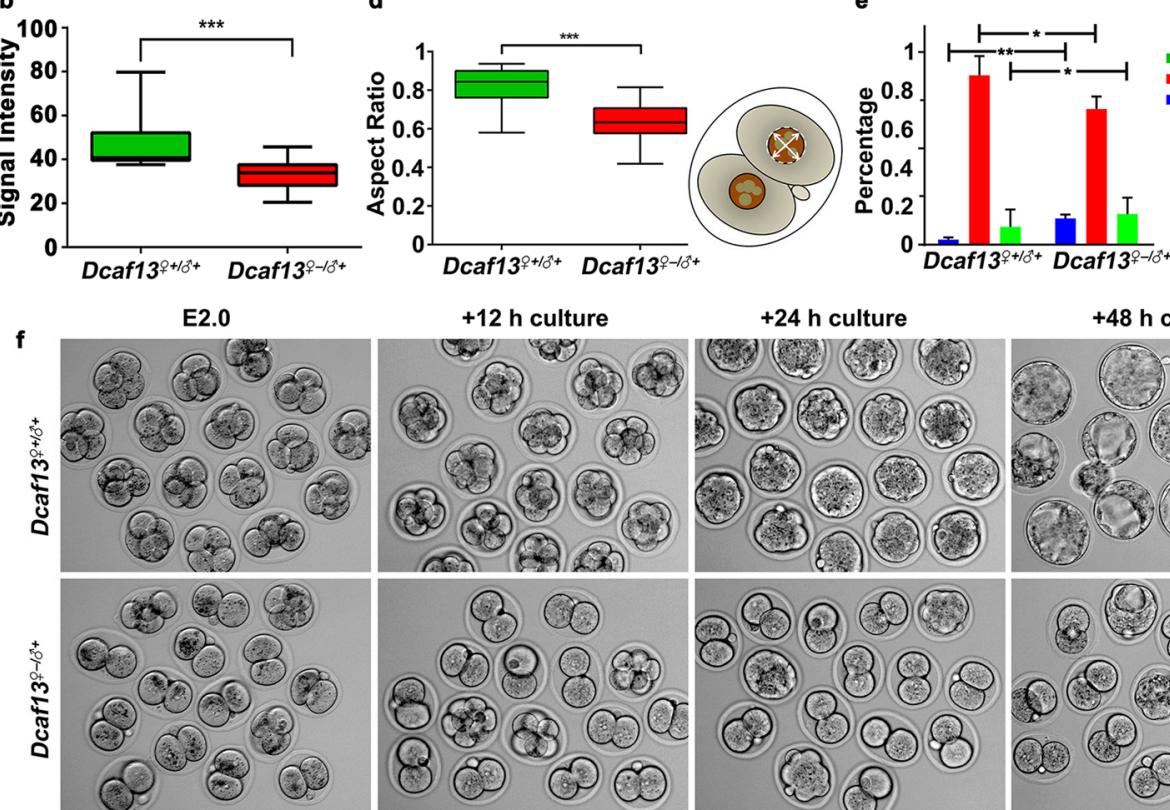

Large $\left(>100 \mathrm{um}^{2}\right)$ -Normal (10-100 um²) - Small $\left(<10 \mathrm{um}^{2}\right)$
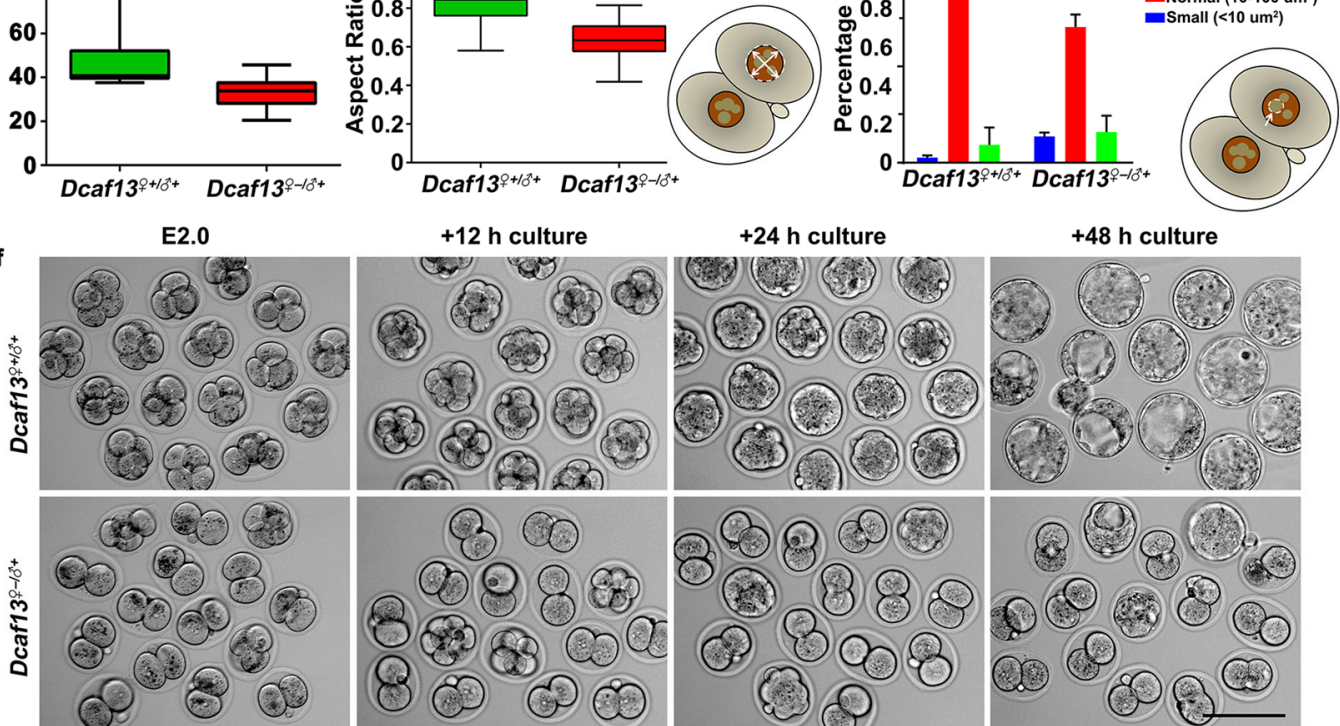

h

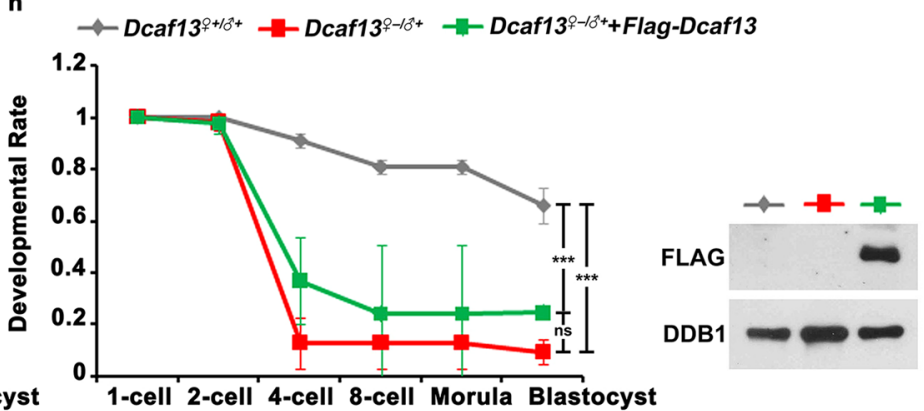

Figure 2. DCAF13-maternally depleted embryos have abnormal chromatin tightness levels and nuclear configuration, and are arrested at the two-cell stage. (a) DCAF13-maternally depleted embryos showed decreased DNase I accessibility. Scale bar, $20 \mu \mathrm{m}$. (b) The positive TUNEL signal of $0.1 \mathrm{U} / \mu \mathrm{L}$ was indicated. $* * *$ to $p<0.001$. Dcaf13 ${ }^{+1+}(\mathrm{n}=12), D c a f 13^{-l+}(\mathrm{n}=20)$. (c) Immunofluorescence using antibodies against B23 (in green) and DNA (in red) in two-cell embryos. The abnormal nuclear morphology (white dotted line) and the abnormal nucleoli (white arrow) were indicated. Scale bar, $20 \mu \mathrm{m}$. Abnormal nuclear morphology was quantified at (d) Classification of nucleoli at (e). ${ }^{*} p<0.05, * * p<0.01$. (f) Representative images for two consecutive days of in vitro culture for Dcaf $13^{+/}$or Dcaf $13^{-1+}$ embryos collected at E2. Scale bar, $100 \mu \mathrm{m}$. (g) Rates of embryo development of (f). (h) The numbers of zygotes, two-cell, four-cell, eight-cell, morula, and blastocysts were counted at each time point (left). Western blot results showed levels of FLAG-DCAF13 in 2-cell embryos with or without mRNA microinjection at the zygote stage. DDB1 was blotted as a loading

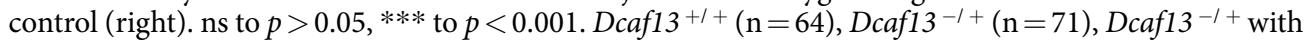
microinjection of FLAG-tagged DCAF13 $(\mathrm{n}=57)$. 


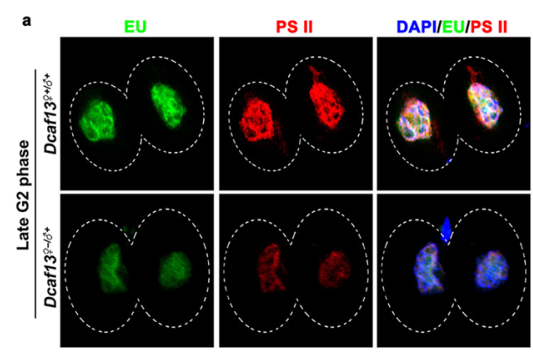

b

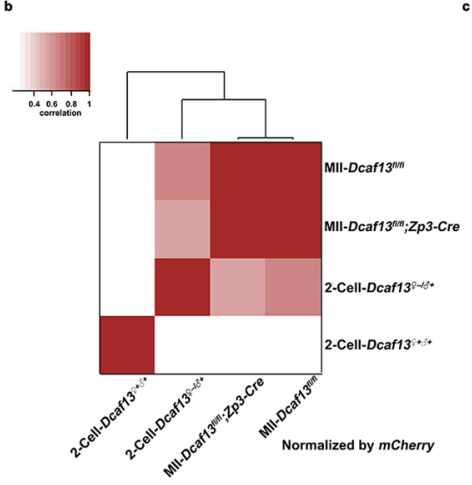

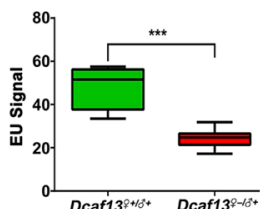

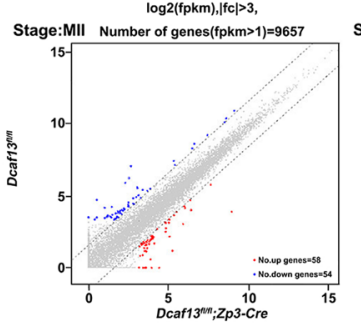

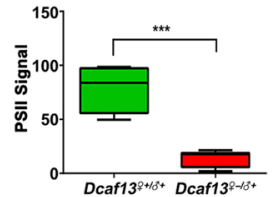

$\log 2(\mathrm{fpkm}),|\mathrm{fc}|>3$,

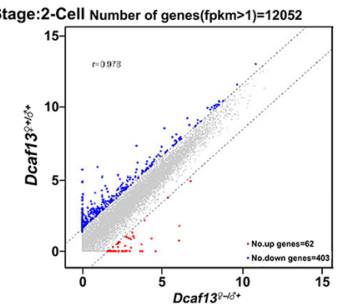

d

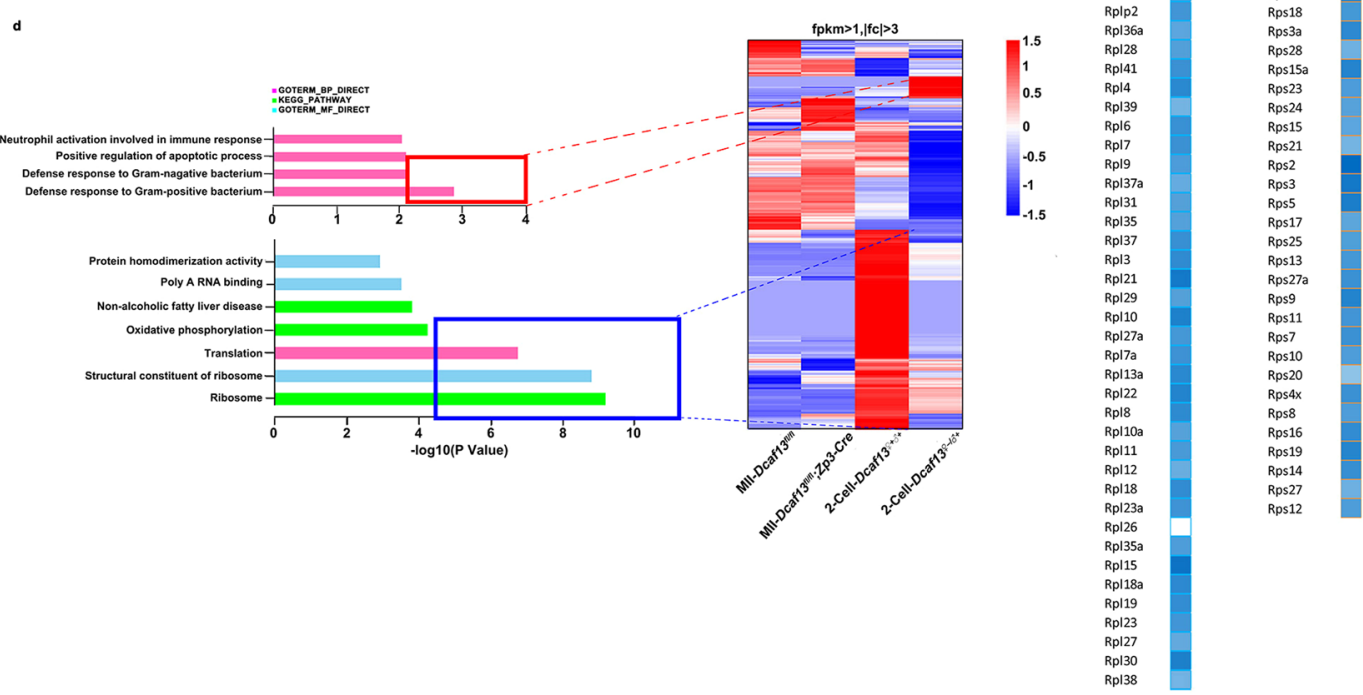

Figure 3. Maternal DCAF13 affects zygotic genome activation by the two-cell stage. (a) Detection of newly synthesized RNA by 5-ethynyl uridine (EU) (in green) incorporation and the activity of polymerase II (POLII) (PSII in red) in two-cell embryos of Dcaf $13^{+1+}$ or Dcaf $13^{-1+}$ is shown (top), and the measurement of the intensity was indicated (bottom). Scale bar, $20 \mu \mathrm{m}$. *** to $p<0.001$. Dcaf $13^{+l+}(\mathrm{n}=10)$, Dcaf $13^{-1+}$ $(\mathrm{n}=10)$. (b) Heat map and cluster tree represented the relative mRNA levels of total transcripts in Dcaf $13^{\mathrm{fl} / \mathrm{fl}}$ and Dcaf $13^{\text {flffl}}$;Zp3-Cre MII oocytes as well as Dcaf $13^{+l+}$ or Dcaf $13^{-1+}$ two-cell embryos. (c) Gene scatter plots showed that transcript levels increasing more than three folds in Dcaf1 $3^{f l f} ; Z$ Z 3 -Cre MII oocytes (Dcaf13 $3^{f l f l} ; Z p 3-C r e / D c a f 13^{f l f l}>3$ ) were upregulated (red); Transcript levels decreasing more than three folds (Dcaf1 $3^{\text {flfl } / D c a f 13^{f l f l} ; Z p 3-C r e}>3$ ) were downregulated (blue) (top). Transcript levels increasing more than three folds in Dcaf $13^{-1+}$ two-cell embryos were upregulated (red); transcript levels decreasing more than three folds in Dcaf $13^{-1+}$ two-cell embryos were downregulated (blue) (bottom). (d) Gene ontology (GO) analysis plot and heat map indicated the function of transcripts whose levels increased (red) or decreased (blue) in Dcaf $13^{-1+}$ two-cell embryos compared to control. (e) Heat map described the level change of transcripts encoding ribosomal proteins grouped by ribosome large and small subunit in Dcaf $13^{-1+}$ two-cell embryos. The fold changes (Dcaf13 $3^{-1+} /$ Dcaf $13^{+l+}$ ) were listed at the top of the boxes from white to blue (from large to small).

we investigated the gene expression profiling by performing RNA sequencing (RNA-seq) for MII oocytes and embryos at the two-cell stage with two biological replicates in a cohort of control and mutant samples $(\mathrm{n}=10$ each). The cDNA libraries were set up, and replicates from control and mutant oocytes as well as embryos were 


\begin{tabular}{|l|l|}
\hline Sample & Mapping ratio \\
\hline MII-Dcaf13 $3^{f / f l}$ & $88.00 \%$ \\
\hline MII-Dcaf13 $3^{f / f} ; Z p 3-C r e$ & $79.30 \%$ \\
\hline 2-cell-Dcaf13//fl & $69.10 \%$ \\
\hline 2-cell-Dcaf13 $3^{f / f} ; Z$ Zp3-Cre & $70.30 \%$ \\
\hline
\end{tabular}

Table 1. The mapping ratio of the RNA-seq samples.

\begin{tabular}{|c|c|c|c|}
\hline \multirow[b]{2}{*}{ Stage } & \multirow[b]{2}{*}{ FPKM } & \multicolumn{2}{|l|}{ Number of genes } \\
\hline & & Dcaf13 ${ }^{f / f} ; Z$ Zp3-Cre & Dcaf13 $3^{f / f l}$ \\
\hline \multirow{5}{*}{ MII } & $\mathrm{FPKM}<=0.1$ & 9583 & 9310 \\
\hline & $0.1<$ FPKM $<=1$ & 3808 & 3899 \\
\hline & $1<$ FPKM $<=3$ & 2187 & 2234 \\
\hline & $3<$ FPKM $<=5$ & 1078 & 1111 \\
\hline & $5<$ FPKM & 5857 & 5999 \\
\hline \multirow{5}{*}{ 2-cell } & FPKM $<=0.1$ & 7045 & 7441 \\
\hline & $0.1<$ FPKM $<=1$ & 4092 & 3686 \\
\hline & $1<$ FPKM $<=3$ & 2710 & 2441 \\
\hline & $3<$ FPKM $<=5$ & 1360 & 1301 \\
\hline & $5<$ FPKM & 7306 & 7644 \\
\hline
\end{tabular}

Table 2. Gene table at certain FPKM threshold.

used (Fig. 3b); we applied Illumina-based deep RNA sequencing on these samples. mCherry mRNA was incorporated in the samples as the reference. And the mapping ratio of the samples were normal (Table 1). We normalized the FPKM values of genes to the FPKM values of $m$ Cherry. According to the FPKM values of genes, we divided transcripts into five groups in MII/two-cell stage (Table 2). We found that DCAF13 deleted in oocytes did not affect MII oocyte transcriptome dramatically, but maternal DCAF13 depletion caused a decrease in global transcription levels at the two-cell stage (Fig. 3b). Our analysis revealed that 62 genes were upregulated, and 403 genes were downregulated in the Dcaf $13^{-1+}$ two-cell embryos compared to the Dcaf $13^{+l+}$ embryos when the gene scattered by fold changes above three. Furthermore, the analysis of MII oocyte transcriptomes also revealed that a few genes were misregulated in DCAF13-deleted oocytes compared to maternal DCAF13-depleted embryos (Fig. 3c). This underlined the specificity of the effects caused by maternal DCAF13 in two-cell embryos.

Gene ontology analysis of up- and downregulated genes at the two-cell stage suggested that the apoptotic process-related genes were upregulated mostly in DCAF13-maternally depleted two-cell embryos, and, surprisingly, ribosome and translation-related genes were downregulated mostly after maternal DCAF13 depletion (Fig. 3d). To further analyze the expression changes of genes encoding ribosomal proteins, we generated the heat map of these genes grouped by ribosome large and small subunits (Fig. 3e). Strikingly, all genes were shown to be downregulated. In line with our previous work, we concluded that maternal DCAF13 deficiency could disorganize the zygotic genome activation.

Absence of maternal DCAF13 abrogates zygotic nucleus function in transcription and results in decreased translation. To further confirm the RNA-seq results, we selected several translation-related genes (Rpl26, Rpl39, Rps20, and Rps21) that should be transcript-activated, so that we could detect their transcription. Because the transcription of many genes were decreased after maternal DCAF13-depetion, exogenous $m$ Cherry mRNA was added to the samples as a control for normalization. Relative mRNA levels of other genes were compared to the levels of $m$ Cherry mRNA in the same sample. It could be concluded that all of the selected genes failed to be expressed at the two-cell stage in DCAF13-maternally depleted embryos compared to the control group (Fig. 4a). This phenotype was more remarkable when the control group developed to the four-cell stage.

Since the nucleolus morphology changed, and B23 staining intensity decreased in DCAF13-maternally depleted two-cell embryos, we hypothesized that the nucleolar function might be affected. The major function of nucleoli is rDNA transcription, and it is essential for the successful development of ZGA ${ }^{16}$. Therefore, we investigated the nucleolar function-related gene expression (pre-rRNA, Ubtf, FBL, and B23). The results showed that those genes could be activated normally in the control along the embryonic development, but their expression was significantly lower in the Dcaf13 ${ }^{-1+}$ group (Fig. 4b). H4K16Ac is a marker for silencing rDNA transcription $^{17}$. We examined the H4K16Ac levels in late two-cell embryos by immunofluorescence, and we found that a remarkable increase occurred in Dcaf $13^{-1+}$ embryos (Fig. 4c). These results revealed that the absence of maternal DCAF13 led to defects in nucleolus function and decrease in rRNA production at the two-cell stage.

To further assess the impact of DCAF13 maternal depletion on the ribosomal protein gene expression and rRNA transcription, we performed HPG staining to detect their translational activity, as they constitute the ribosome. After measuring the HPG staining intensity, as we predicted, a significant decrease was detected in Dcaf $13^{-1+}$ late two-cell embryos (Fig. 4d). 

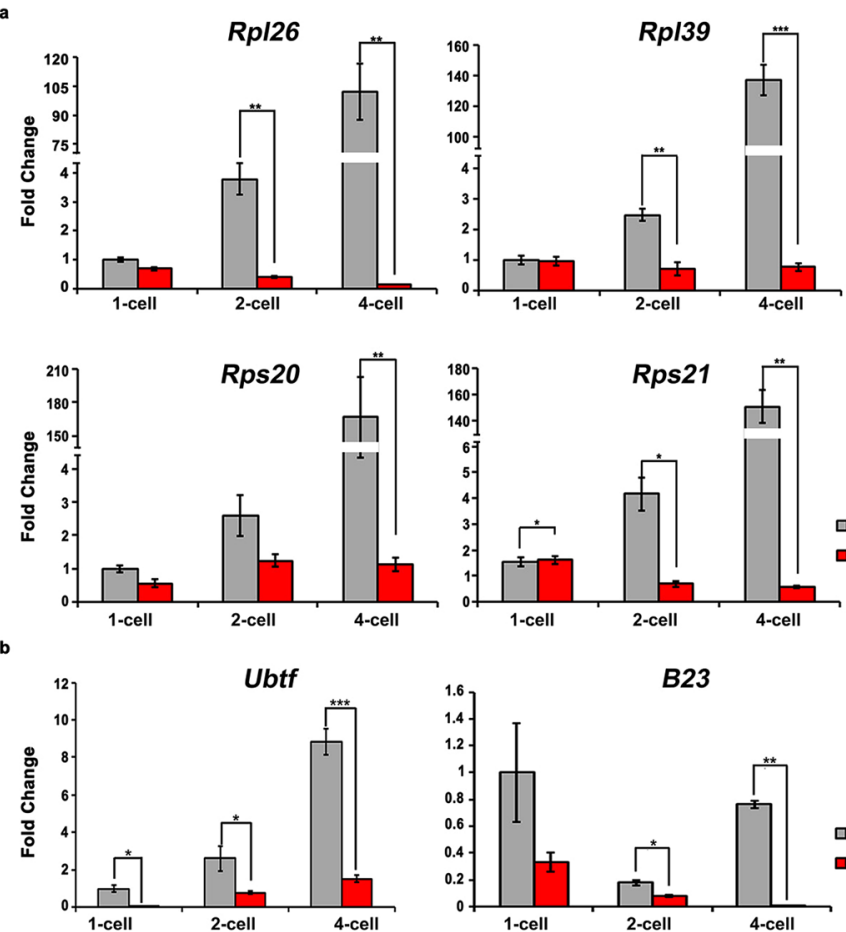

FBL
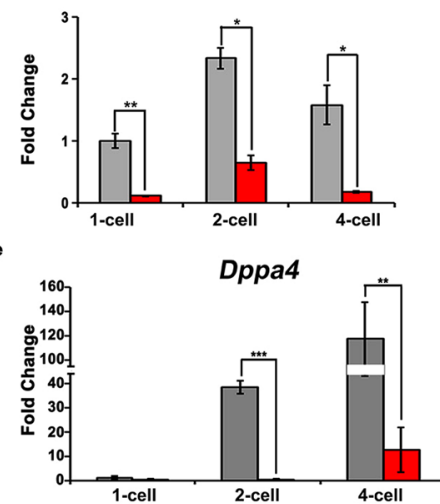
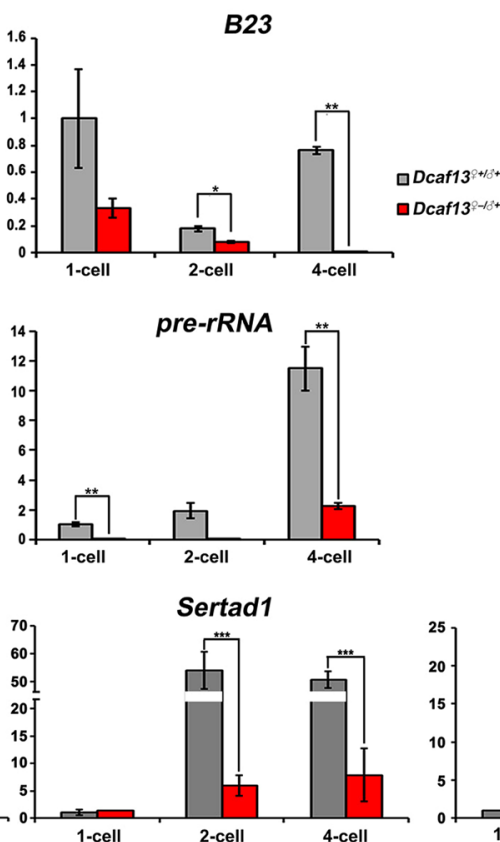
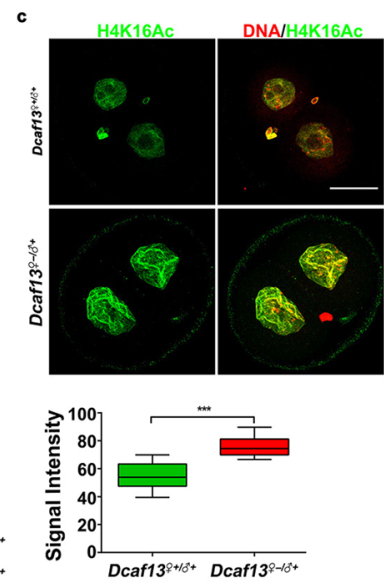

d
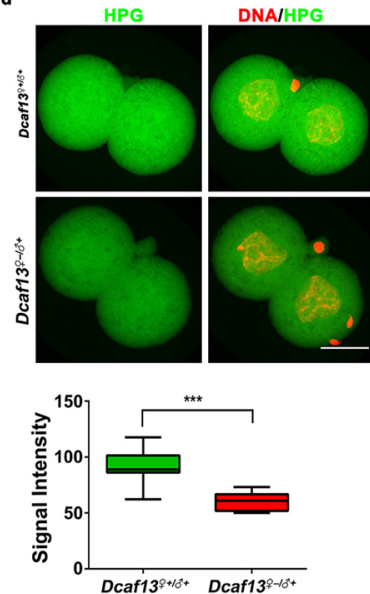

Ppp1r8

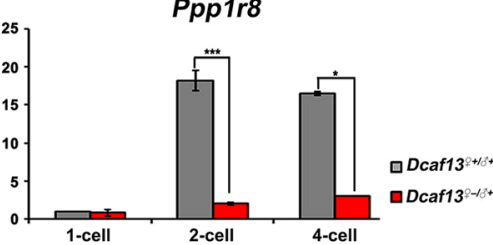

Figure 4. Absence of maternal DCAF13 abrogates zygotic nucleus function in transcription and results in decreased translation. (a,b) Graphical representation of the mean expression level \pm standard error of the mean (SEM) by reverse transcription polymerase chain reaction (RT-PCR) for several ribosomal protein genes (a) and rDNA processing factor gene (b) in Dcaf13 ${ }^{+1}$ (in gray) or Dcaf $13^{-1+}$ (in red) zygotes, two-cell and four-cell (twocell stage in Dcaf1 $3^{-1+}$ ) embryos. $* p<0.05$, ** to $p<0.01$ and $* * *$ to $p<0.001$. (c) Immunofluorescence using antibodies against $\mathrm{H} 4 \mathrm{~K} 16 \mathrm{Ac}$ (in green) and DNA (in red) in two-cell embryos (top), and intensity measurement

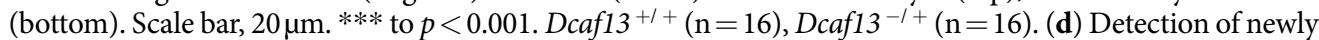
synthesized protein by homopropargylglycine (HPG) incorporation in two-cell embryos of Dcaf $13^{+1+}$ or Dcaf $13^{-1+}$ was shown (top), also with the intensity measurement (bottom). Scale bar, $20 \mu \mathrm{m}$. *** to $p<0.001$. Dcaf13 $3^{+l+}(\mathrm{n}=14)$, Dcaf13 ${ }^{-1+}(\mathrm{n}=14)$. (e) Graphical representation of the mean expression level \pm SEM by RTPCR for several representative zygotic genes in Dcaf $13^{+/+}$(in gray) or Dcaf $13^{-1+}$ (in red) zygotes, two-cell and four-cell (two-cell stage in Dcaf13 ${ }^{-1+}$ ) embryos. $* p<0.05$, ** to $p<0.01$, and $* * *$ to $p<0.001$.

These results indicated that zygotic genome activation in $D c a f 13^{-1+}$ embryos was impaired. We also detected several representative zygotic genes that were highly transcribed in two-cell embryos ${ }^{18}$. Notably, all these genes were downregulated in Dcaf $13^{-1+}$ two-cell embryos (Fig. 4e). This indicated that maternal DCAF13 was required for extensive zygotic gene transcription and not only for translation-related genes.

\section{Discussion}

In mouse embryos, after fertilization, the first major developmental transition is the $\mathrm{MZT}^{9}$. The development depends on maternal protein and RNA stored by oocytes before ZGA, which occurs at the one-cell stage $^{15}$. The MZT is the opening that embryos take charge of gene expression to control cell differentiation and further development instead of maternal origin. After fertilization, decondensation and reorganization 


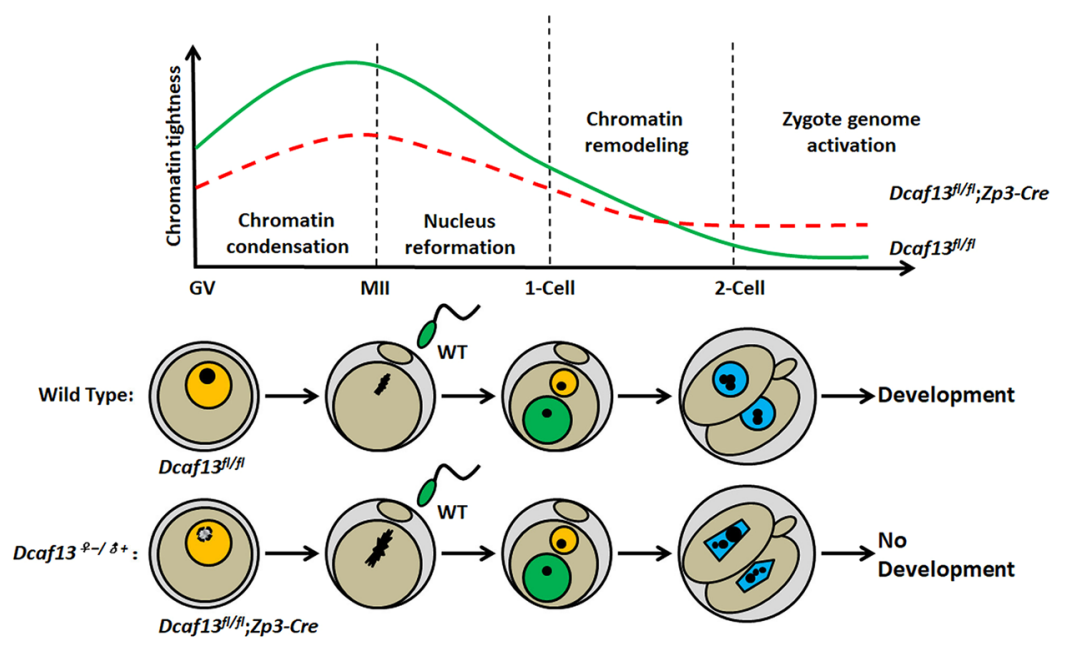

Figure 5. Schematic model for the role of maternal DCAF13 during the maternal-zygotic transition. In oocytes, the chromatin is condensed during NSN-SN transition, and is further condensed during meiotic maturation. After fertilization, chromatin structure turns to be extremely loose in pronuclei, leading to the whole genome started to be transcribed. However, the DCAF13-deleted oocytes have defects of chromatin tightening, as well as insufficient chromatin loosening after fertilization. Therefore, DCAF13 in growing oocyte is required for the compaction of chromatin during NSN-SN transition. Meanwhile, the correctly compacted genomic DNA in oocyte is also a prerequisite for embryonic chromatin loosening and nucleus reformation during MZT.

of chromatin in male and female gametes is a crucial step needed for embryonic development ${ }^{19}$. In addition, the factors contributing to nucleus reformation before ZGA are strictly of maternal origin ${ }^{8}$. In this study, we investigated the critical factor contributing to zygotic nucleus reformation, and we identified DCAF13, a maternal protein located in the nucleoli of growing oocyte that played an important maternal role in zygotic nucleus reformation and was required for ZGA. Although DCAF13 expression is low in zygote and 2-cell embryos, maternal deletion of DCAF13 in growing oocytes still causes developmental arrest at this stage. So we selected this interesting model to elucidate the function of maternal DCAF13 during the early development of the zygotes. We showed that maternal DCAF13 was a major regulator of the chromatin tightness during MZT, especially for ribosomal protein gene transcription (as shown from RNA-seq data) during development. We believe that the MZT defect caused by DCAF13 deletion in oocytes was originated from the growing stage. Even if we supplied exogenous DCAF13 in fully grown GV stage oocytes, there was little chance for us to observe a rescuing effect.

We showed that maternal DCAF13 was essential for chromatin tightness regulation during MZT. DCAF13 deletion in oocytes resulted in loose chromatin, and this influences nucleus reformation and caused chromatin condensation, which destroyed nucleus function. This prevented the development of embryos beyond the two-cell stage (Fig. 5). DCAF13-deleted oocytes possessed potential effects which were not vital for oocyte maturation but fatal for embryo development. In oocytes, the chromatin is condensed during NSN-SN transition, and is further condensed during meiotic maturation. After fertilization, the reverse process occurs. Chromatin structure turns to be extremely loose in pronuclei, leading to the whole genome started to be transcribed. The loosened chromatin structure is involved in their totipotency and high transcription activity. So the chromatin structure closely relates to transcription activity.

We observed defects of chromatin tightening (increased susceptibility of genomic DNA to DNase I digestion) in DCAF13-deleted oocytes, as well as insufficient chromatin loosening after fertilization. Based on the data presented in this manuscript, we proposed that DCAF13 in growing oocyte was required for the compaction of chromatin during NSN-SN transition. Meanwhile, the correctly compacted genomic DNA in oocyte is also a prerequisite for embryonic chromatin loosening and nucleus reformation during MZT. Maternal DCAF13 was the key factor in this process. DCAF13-dependent chromatin tightening in oocyte was required for appropriate transcription activation of early zygotic genes, particular those encoding rRNAs and ribosome proteins.

Reproductive aging is related to the decline in fertility, and endocrine functions draw public attention as women are globally delaying childbearing ${ }^{20}$. It is reported that the quality and developmental potentiality of oocytes are related to age, affecting the MZT and leading to embryonic development failure ${ }^{21}$. Moreover, a dataset of quantitative mass spectrometry of oocytes from young and aged female mice shows that DCAF13 protein is reduced in aged oocytes ${ }^{22}$. The reduction of DCAF13 influences oocyte competence, which is tightly connected to embryonic development ${ }^{12}$. Thus, our study provides an explanation of the reason why menopausal women are prone to miscarriage. 


\begin{abstract}
Methods
Mice. WT C57/B6 mice were obtained from the Shanghai SLAC laboratory, China. Dcaf1 $3^{\text {flfl }}$ and Zp3-Cre mice exhibited a C57BL/6 background. The experimental procedures, and experimental protocols involving mice were approved by the Zhejiang University Institutional Animal Care and Research Committee (Approval \# ZJU20170014), and mouse care and use were performed in accordance with the relevant guidelines and regulations.
\end{abstract}

Western blot analysis. Zygotes were lysed with SDS sample buffer (100 zygotes per sample) and heated for $5 \mathrm{~min}$ at $95^{\circ} \mathrm{C}$. Total zygotes proteins were separated by SDS-PAGE and electrophoretically transferred to PVDF membranes (Millipore, USA), followed by blocking in TBST containing $5 \%$ defatted milk (BD, USA) for 30 min. First, the membranes were incubated with primary antibodies overnight at $4{ }^{\circ} \mathrm{C}$. Then, the membranes were washed in TBST, and incubated with a HRP-linked secondary antibody for $1 \mathrm{~h}$ at room temperature, followed by washing with TBST three times. Finally, bounding antibodies were detected using SuperSignal WestFemto maximum sensitivity substrate (Thermo Fisher, USA). The primary antibodies used and dilution factors are anti-DDB1 (Epitomics, \#3821-1, 1:10000) and anti-FLAG antibodies (Sigma, \#F3165, 1:3000). The secondary antibody was HRP-conjugated anti-rabbit/mouse IgG (Jackson ImmunoResearch Laboratories).

Immunofluorescent microscopy. Oocytes or embryos were fixed in phosphate-buffered saline-buffered $4 \%$ paraformaldehyde (PFA) and penetrated with $0.5 \%$ Triton X-100 (Sangon Biotech, China). They were blocked with $1 \%$ bovine serum albumin (BSA) (Sangon Biotech, China) in PBS. They were incubated with primary antibodies diluted in blocking solution. Next, they were washed in PBS, and labeled with secondary antibodies, counterstained with 40,6-diamidino-2-phenylindole (DAPI) (Sigma-Aldrich). Finally, they were mounted on glass slides using SlowFade Gold Antifade Reagent (Life Technologies). Imaging was performed on a Zeiss LSM710 confocal microscope. Single frame of each was scanned at the middle focal plane of their nucleus. Semi-quantitative analysis of the fluorescence signals was conducted using the NIH Image program ImageJ. Briefly, the pixel value/unit area was measured for the nucleus, and the value for the cytoplasm was subtracted as background. The value obtained was multiplied by the nuclear area to yield the total amount of fluorescence in the nucleus.

The primary antibodies used were anti-H4K16Ac (Abcam, ab109463, 1:400), anti-B23 (Abcam, ab10530, 1:1000). The secondary antibodies were Alexa Fluor 594-conjugated goat anti-rabbit/mouse IgG (Life Technology) and Alexa Fluor 488-conjugated goat anti-rabbit/mouse IgG (Jackson ImmunoResearch Laboratories, USA).

In vitro transcription and preparation of mRNAs for microinjections. Expression vectors were linearized and subjected to phenol/chloroform extraction and ethanol precipitation. The linearized DNAs were in vitro transcribed using the SP6 message mMACHINE kit (Invitrogen, AM1340). Transcribed mRNAs were added with poly(A) tails using the mMACHINE kit (Invitrogen, AM1350) and were recovered by lithium chloride precipitation and resuspended in nuclease-free water.

Superovulation and fertilization. Female mice at the pubertal stage (21-23 day old) were intraperitoneally injected with 5 IU of pregnant mare serum gonadotropin (PMSG, Ningbo Sansheng Pharmaceutical Co., ltd., P.R China). After $44 \mathrm{~h}$, these mice were then injected with $5 \mathrm{IU}$ of human chorionic gonadotropin (hCG, Ningbo Sansheng Pharmaceutical Co., ltd., P.R China). Immediately after hCG injection, female mice were mated with 10-12-week-old WT males that were known to be fertile. Successful coitus was confirmed by the presence of vaginal plugs the next morning. Preimplantation embryos were collected from oviducts at the indicated times after hCG injection.

Microinjection of zygotes. For microinjection, mouse zygotes were harvested in M2 medium at $24 \mathrm{~h}$ after hCG injection. Microinjections were performed using an Eppendorf transferman NK2 micromanipulator. Each zygote was microinjected with approximately 5 to $10 \mathrm{pL}$ samples. Microinjected zygotes were then washed with $\mathrm{M} 2$ and cultured in prewarmed $\mathrm{KSOM}$ medium at $37^{\circ} \mathrm{C}$ with $5 \% \mathrm{CO}_{2}$.

TUNEL assay for detection of DNase I sensitivity. $\quad$ Dcaf $13^{f l f l}$ and Dcaf $13^{\text {flffl}} ; Z p 3-C r e$ GV oocytes or embryos were collected as described above and pre-extracted immediately in ice-cold solution $(50 \mathrm{mM} \mathrm{NaCl}$, $3 \mathrm{mM} \mathrm{MgCl}_{2}, 0.5 \%$ Triton X-100, and $300 \mathrm{mM}$ sucrose in $25 \mathrm{mM}$ 4-(2-hydroxyethyl)-1-piperazineethanesulfonic acid (HEPES), $\mathrm{pH} 7.4$ ) for $5 \mathrm{~min}$. The oocytes were incubated with different concentrations of DNase 1 (NEB) for $5 \mathrm{~min}$ at $37^{\circ} \mathrm{C}$ in the same buffer without Triton X-100 and fixed for $10 \mathrm{~min}$ in $2 \%$ PFA/PBS at room temperature. TUNEL assay was performed using Click-iT TUNEL Alexa Fluor Imaging Assay (Life Technologies, C10245) according to the manufacturer's instructions ${ }^{23}$.

5-Ethynyl uridine (EU) staining and homopropargylglycine (HPG) staining. For EU staining, oocytes were incubated for $1 \mathrm{~h}$ with $100 \mathrm{mM}$ EU from the Click-iT RNA Alexa Fluor 488 Imaging kit (Thermo Fisher Scientific). Oocytes were then fixed using 4\% PFA/PBS for $15 \mathrm{~min}$ and permeabilized with $0.25 \%$ Triton/ PBS for $15 \mathrm{~min}$. The Click-It reaction was performed according to the manufacturer's instructions. Oocytes were then counterstained with DAPI for 15 min before the slides were mounted with SlowFade Gold Antifade Reagent and imaged on a Zeiss LSM 710 at $63 \mathrm{X}$. EU signal intensity was calculated using Image ${ }^{24}$. The synthesis of protein was measured using Click-iT HPG Alexa Fluor Protein Synthesis Assay kits (Thermo Fisher Scientific) according the manufacturer's instructions. Oocytes were treated with the Click-iT HPG reagent and then fixed with $4 \%$ PFA. Oocytes were permeated with $0.5 \%$ Triton X-100, and Alexa Fluor 488 was then conjugated to the protein using click chemistry. Fluorescence signal was detected using a Zeiss LSM 710 microscope and quantified using ImageJ. 
RNA-seq library preparation. Oocytes and embryos were collected from indicated genotypes (10 oocytes or embryos per sample). Each sample was added with $4 \mu \mathrm{l}$ lysis buffer $(0.2 \%$ Triton X-100, RNase inhibitor, dNTPs, oligo-dT primers and 100 pg mCherry mRNA spike-in) and immediately processed into cDNA using Smart-seq2 as described previously ${ }^{25}$. Sequencing libraries were constructed from $500 \mathrm{pg}$ of pre-amplified cDNA using a DNA library preparation kit (TruePrep DNA Library Prep Kit V2 for Illumina, Vazyme) which based on Tn5 transposase. Barcoded libraries were pooled at equimolar ratios and sequenced on illumina platform with 150 bp pair-end reads.

RNA-seq data analysis. RNA-seq was performed with biological replicates for all samples. RNA-seq reads were sequenced on the Illumina HiSeq platform as paired end 150-base reads. Raw reads were trimmed with Trimmomatic- 0.36 to $50 \mathrm{bp}$ and mapped to the mouse genome $(\mathrm{mm} 9)$ with TopHat (v2.0.11). The mapped reads were subsequently assembled into transcripts guided by reference annotation (University of California at Santa Cruz [UCSC] gene models) with Cufflinks version 2.2.1. The expression level of each transcript was quantified with normalized FPKM based on the FPKM of exogenous $m$ Cherry. Differentially expressed genes were identified by asking for a fold change $>10$ with FPKM $>1$ in at least one sample. GO analysis for enrichment of GEGs (differentially expressed genes) was determined using the Database for Annotation, Visualization and Integrated Discovery (DAVID).

Statistical analyses were implemented with R (http://www.rproject.org). A Spearman's R coefficient was calculated using the cor function with default parameters and complete method was used to cluster the genes.

RNA isolation and reverse transcription polymerase chain reaction (RT-PCR). Total RNA was extracted using RNeasy Mini kit (Qiagen, 74106). RT-PCR analysis was performed using the Power SYBR Green PCR Master Mix (Applied Biosystems, Life Technologies) and an Applied Biosystems 7500 Real-Time PCR System. Relative mRNA levels were normalized to the levels of exogenous $m$ Cherry mRNA (external control). The final concentration of $m$ Cherry in sample was $0.001 \mathrm{ng} / \mu \mathrm{L}$. The relative transcript levels of samples were compared to the control, and the fold-changes were shown. Each experiment was conducted with three biological replicates.

Statistical analysis. Results were expressed as means \pm standard error of the mean (SEM). Most experiments included at least three independent samples and were repeated at least three times. Results for two experimental groups were compared by two-tailed unpaired Student's $t$-tests. Statistically significant values of $p<0.05$, $p<0.01$, and $p<0.001$ by two-tailed Student's $t$-test were indicated by asterisks $(*),(* *)$, and (***) respectively. "ns" indicates non-significant.

The box and whiskers graphs in the manuscript were generated by Graphpad 6.0 (Graphpad Software Inc. USA). The box extended from the $25^{\text {th }}$ to $75^{\text {th }}$ percentiles. The whiskers went down to the smallest value and up to the largest. We have added more details about data processing in the method part.

\section{Data Availability}

The dataset generated and/or analysed during the current study is available from the corresponding author on reasonable request.

\section{References}

1. Yartseva, V. \& Giraldez, A. J. The Maternal-to-Zygotic Transition During Vertebrate Development: A Model for Reprogramming. Current topics in developmental biology 113, 191-232, https://doi.org/10.1016/bs.ctdb.2015.07.020 (2015).

2. Li, L., Lu, X. \& Dean, J. The maternal to zygotic transition in mammals. Mol Aspects Med 34, 919-938, https://doi.org/10.1016/j. mam.2013.01.003

3. Yu, C. et al. BTG4 is a meiotic cell cycle-coupled maternal-zygotic-transition licensing factor in oocytes. Nat Struct Mol Biol 23, 387-394, https://doi.org/10.1038/nsmb.3204

4. Flach, G., Johnson, M. H., Braude, P. R., Taylor, R. A. \& Bolton, V. N. The transition from maternal to embryonic control in the 2-cell mouse embryo. The EMBO journal 1, 681-686 (1982).

5. Borsos, M. \& Torres-Padilla, M. E. Building up the nucleus: nuclear organization in the establishment of totipotency and pluripotency during mammalian development. Genes \& development 30, 611-621, https://doi.org/10.1101/gad.273805.115 (2016).

6. Inoue, A., Nakajima, R., Nagata, M. \& Aoki, F. Contribution of the oocyte nucleus and cytoplasm to the determination of meiotic and developmental competence in mice. Human reproduction 23, 1377-1384, https://doi.org/10.1093/humrep/den096 (2008).

7. Liu, N. et al. Pyruvate prevents aging of mouse oocytes. Reproduction 138, 223-234, https://doi.org/10.1530/REP-09-0122 (2009).

8. Ooga, M., Fulka, H., Hashimoto, S., Suzuki, M. G. \& Aoki, F. Analysis of chromatin structure in mouse preimplantation embryos by fluorescent recovery after photobleaching. Epigenetics-Us 11, 85-94, https://doi.org/10.1080/15592294.2015.1136774 (2016).

9. Lee, M. T., Bonneau, A. R. \& Giraldez, A. J. Zygotic genome activation during the maternal-to-zygotic transition. Annual review of cell and developmental biology 30, 581-613, https://doi.org/10.1146/annurev-cellbio-100913-013027 (2014).

10. Kyogoku, H., Kitajima, T. S. \& Miyano, T. Nucleolus precursor body (NPB): a distinct structure in mammalian oocytes and zygotes. Nucleus 5, 493-498, https://doi.org/10.4161/19491034.2014.990858 (2014).

11. Lee, J. \& Zhou, P. DCAFs, the missing link of the CUL4-DDB1 ubiquitin ligase. Mol Cell 26, 775-780, https://doi.org/10.1016/j. molcel.2007.06.001 (2007).

12. Zhang, Y. L. et al. DCAF13 promotes pluripotency by negatively regulating SUV39H1 stability during early embryonic development. The EMBO journal 37, https://doi.org/10.15252/embj.201898981 (2018).

13. Zhang, J. et al. Mammalian nucleolar protein DCAF13 is essential for ovarian follicle maintenance and oocyte growth by mediating rRNA processing. Cell death and differentiation, https://doi.org/10.1038/s41418-018-0203-7 (2018).

14. de Vries, W. N. et al. Expression of Cre recombinase in mouse oocytes: a means to study maternal effect genes. Genesis 26, 110-112 (2000).

15. Jukam, D., Shariati, S. A. M. \& Skotheim, J. M. Zygotic Genome Activation in Vertebrates. Developmental cell 42, 316-332, https:// doi.org/10.1016/j.devcel.2017.07.026 (2017).

16. Heyn, P., Salmonowicz, H., Rodenfels, J. \& Neugebauer, K. M. Activation of transcription enforces the formation of distinct nuclear bodies in zebrafish embryos. RNA biology 14, 752-760, https://doi.org/10.1080/15476286.2016.1255397 (2017).

17. Zhou, Y. \& Grummt, I. The PHD finger/bromodomain of NoRC interacts with acetylated histone H4K16 and is sufficient for rDNA silencing. Current biology: CB 15, 1434-1438, https://doi.org/10.1016/j.cub.2005.06.057 (2005). 
18. Yu, C. et al. BTG4 is a meiotic cell cycle-coupled maternal-zygotic transition licensing factor in oocytes. Nature Structural \& Molecular Biology 23, 387-394, https://doi.org/10.1038/nsmb.3204 (2016).

19. Burns, K. H. et al. Roles of NPM2 in chromatin and nucleolar organization in oocytes and embryos. Science 300, 633-636, https:// doi.org/10.1126/science.1081813 (2003).

20. Heffner, L. J. Advanced maternal age-how old is too old? The New England journal of medicine 351, 1927-1929, https://doi. org/10.1056/NEJMp048087 (2004).

21. Schwarzer, C. et al. Maternal age effect on mouse oocytes: new biological insight from proteomic analysis. Reproduction 148, 55-72, https://doi.org/10.1530/REP-14-0126 (2014).

22. Wang, B. Y., Pfeiffer, M. J., Schwarzer, C., Arauzo-Bravo, M. J. \& Boiani, M. DNA Replication Is an Integral Part of the Mouse Oocyte's Reprogramming Machinery. Plos One 9, https://doi.org/10.1371/journal.pone.0097199 (2014).

23. Nashun, B. et al. Continuous Histone Replacement by Hira Is Essential for Normal Transcriptional Regulation and De Novo DNA Methylation during Mouse Oogenesis. Mol Cell 60, 611-625, https://doi.org/10.1016/j.molcel.2015.10.010

24. Collins, T. J. ImageJ for microscopy. BioTechniques 43, 25-30, https://doi.org/10.2144/000112517 (2007).

25. Picelli, S. et al. Full-length RNA-seq from single cells using Smart-seq. 2. Nat Protoc 9, 171-181, https://doi.org/10.1038/ nprot.2014.006 (2014).

\section{Acknowledgements}

This study is funded by the National Natural Science Foundation of China (81871415 and 31371449 ).

\section{Author Contributions}

Y.J., J.-L.S. and H.Y.F. designed the study and revised the manuscript. Y.L. and L.W.Z. performed the experiments and data analysis. L.W.Z. wrote the manuscript. H.Y.F. and Y.J. revised the manuscript.

\section{Additional Information}

Supplementary information accompanies this paper at https://doi.org/10.1038/s41598-019-42179-w.

Competing Interests: The authors declare no competing interests.

Publisher's note: Springer Nature remains neutral with regard to jurisdictional claims in published maps and institutional affiliations.

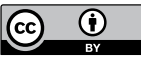

Open Access This article is licensed under a Creative Commons Attribution 4.0 International License, which permits use, sharing, adaptation, distribution and reproduction in any medium or format, as long as you give appropriate credit to the original author(s) and the source, provide a link to the Creative Commons license, and indicate if changes were made. The images or other third party material in this article are included in the article's Creative Commons license, unless indicated otherwise in a credit line to the material. If material is not included in the article's Creative Commons license and your intended use is not permitted by statutory regulation or exceeds the permitted use, you will need to obtain permission directly from the copyright holder. To view a copy of this license, visit http://creativecommons.org/licenses/by/4.0/.

(C) The Author(s) 2019 